\title{
Simulated Field Maps for Susceptibility Artefact Correction in Interventional MRI
}

\author{
Martin Kochan - Pankaj Daga - Ninon Burgos - Mark White • \\ M. Jorge Cardoso - Laura Mancini - Gavin P. Winston . \\ Andrew W. McEvoy • John Thornton · Tarek Yousry • John S. Duncan • \\ Danail Stoyanov · Sébastien Ourselin
}

Received: date / Accepted: date

\begin{abstract}
Purpose Intraoperative MRI (iMRI) is a powerful modality for acquiring images of the brain to facilitate precise image guided neurosurgery. Diffusion-weighted MRI (DW-MRI) provides critical information about location, orientation and structure of nerve fibre tracts, but suffers from the "susceptibility artefact" stemming from magnetic field perturbations due to the step change in magnetic susceptibility at air-tissue boundaries in the head. An existing approach to correct the artefact is to acquire a field map by means of an ad-

This work was supported by the UCL Doctoral Training Programme in Medical and Biomedical Imaging studentship funded by the EPSRC. Danail Stoyanov would like to thank for the support of The Royal Academy of Engineering/EPSRC Research Fellowship. Sébastien Ourselin receives funding from the EPSRC (EP/H046410/1, $\mathrm{EP} / \mathrm{J} 020990 / 1, \mathrm{EP} / \mathrm{K} 005278)$, the MRC (MR/J01107X/1), the EU-FP7 project VPH-DARE@IT (FP7-ICT-2011-9601055), the NIHR Biomedical Research Unit (Dementia) at UCL and the National Institute for Health Research University College London Hospitals Biomedical Research Centre (NIHR BRC UCLH/UCL High Impact Initiative).
\end{abstract}

Martin Kochan (ه) . Pankaj Daga · Ninon Burgos . M. Jorge Cardoso · Danail Stoyanov · Sébastien Ourselin

Centre for Medical Image Computing, University College

London, London, UK

E-mail: m.kochan.12@ucl.ac.uk

Mark White · Laura Mancini · Andrew W. McEvoy · John Thornton - Tarek Yousry

National Hospital for Neurology and Neurosurgery, UCLH NHS Foundation Trust, London, UK

M. Jorge Cardoso · Sébastien Ourselin

Dementia Research Centre, Institute of Neurology, University College London, London, UK

Gavin P. Winston · John S. Duncan

Department of Clinical and Experimental Epilepsy, Institute of Neurology, University College London, London, UK ditional MRI scan. However, to recover true field maps from the acquired field maps near air-tissue boundaries is challenging and acquired field maps are unavailable in historical MRI datasets. This paper reports a detailed account of our method to simulate field maps from structural MRI scans that was first presented at IPCAI 2014 and provides a thorough experimental and analysis section to quantitatively validate our technique.

Methods We perform automatic air-tissue segmentation of intraoperative MRI scans, feed the segmentation into a field map simulation step and apply the acquired and the simulated field maps to correct DW-MRI datasets. Results We report results for 12 patient datasets acquired during anterior temporal lobe resection surgery for the surgical management of focal epilepsy. We find a close agreement between acquired and simulated field maps and observe a statistically significant reduction of the susceptibility artefact in DW-MRI datasets corrected using simulated field maps in the vicinity of the resection. The artefact reduction obtained using acquired field maps remains better than that using the simulated field maps in all evaluated regions of the brain.

Conclusions The proposed simulated field maps facilitate susceptibility artefact reduction near the resection. Accurate air-tissue segmentation is key to achieving accurate simulation. The proposed simulation approach is adaptable to different iMRI and neurosurgical applications.

Keywords Image guided neurosurgery · Interventional MRI · Magnetic field inhomogeneity · Tissue segmentation · Field map simulation 


\section{Introduction}

Anterior temporal lobe resection (ATLR) is an established treatment for refractory temporal lobe epilepsy [22]. The optic radiation is a fibre tract that conveys visual information from the lateral geniculate nucleus in the thalamus to the primary visual cortex. The fibres representing the superior visual field (Meyer's loop) are vulnerable to damage during ALTR, which can lead to postoperative contralateral superior visual field deficit precluding the seizure-free patient from returning to regular activity, such as through loss of eligibility for driving [5].

Image-guided neurosurgery is an established surgical specialization that involves the display of surgical plans overlaid onto the intraoperative model of brain anatomy to facilitate a potential improvement to the precision of pathological tissue resection and a potential reduction of damage to the surrounding areas such as the optic radiation. The accurate mapping of the preoperative image space to the intraoperative anatomical space is complicated by intraoperative brain shifts that can reach up to $11 \mathrm{~mm}$ [5]. Interventional MRI (iMRI) can provide accurate volumetric information about the intraoperative anatomical space. Diffusionweighted MRI (DW-MRI) provides information about the location, orientation and structure of nerve fibre tracts and can reveal the anterior extent of the optic radiation that varies between subjects [18].

However, DW-MRI is acquired using the echo planar imaging (EPI) pulse sequence, which suffers from severe geometric distortion due to field inhomogeneities i.e. local differences of the $B_{0}$ magnetic field from the nominal field strength. MRI encodes each imaging dimension by superimposing a linear gradient field onto the main field. This linearity is assumed during image reconstruction and thus field inhomogeneities virtually displace the signal origin. EPI has a limited signal bandwidth in the phase-encode (PE) imaging dimension and local inhomogeneities even on the order of ppm result in a geometric distortion along the $\mathrm{PE}$ dimension. $B_{0}$ inhomogeneities due to design constraints on the magnet can be reduced (shimmed) to several ppm by means of superconducting shim coils [6]. Another source of $B_{0}$ inhomogeneities is the perturbation of the magnetic field by non-uniform geometric distribution of magnetic susceptibility in the imaged volume, which is largest near air-tissue boundaries such as the resection cavity, the paranasal sinuses and the petrous part of the temporal bone [14]. This perturbation is shimmed using a set of room-temperature shim coils. However, the residual inhomogeneities due to higher-order perturbations give rise to the susceptiblity artefact, which is char- acterized by severe non-linear geometric warping and intensity distortion, and signal dropout that reduces the signal-to-noise ratio (SNR). These effects complicate DW-MRI processing such as tensor fitting [15].

One approach to correcting the susceptibility distortion artefact is to acquire a residual $B_{0}$ inhomogeneity map ("field map"), requiring approximately 1 minute additional acquisition time and to correct the EPI image by considering the field map as defining linear displacement along the PE dimension. This approach was recently used in a clinical iMRI study and facilitated a reduction of the intraoperative susceptibility artefact [7]. The acquisition process uses the gradientrecalled echo pulse sequence [14], which measures the spin phase evolution between a pair of echoes. The resulting phase-difference map ("acquired field map") is modulo- $2 \pi$ wrapped due to the unknown number of elapsed revolutions. Techniques for the necessary phaseunwrapping to recover the true continuous field map are presented e.g. in [12] and [7]. However, an accurate estimation of the true field map becomes difficult near the resection air-tissue boundary. Firstly, acquired field maps also suffer from low SNR that drops further near the resection due to signal dropout. Secondly, the spatial gradient in the true field map is highest near air-tissue boundaries, which leads to abrupt changes in the observed field map. This presents a challenge to the phase-unwrapping methods that necessarily include a field smoothness regularization constraint and potentially fail to fit the true field map.

A second distortion correction approach is to nonlinearly register the conventional non-EPI T2-weighted (T2w) structural scan with the "b0" image (b0-DWMRI), which is a T2w EPI image acquired without the diffusion gradients, and to correct the diffusionweighted EPI images by applying the resulting deformation. Such correction has been shown to reduce tensor fitting errors in DW-MRI datasets [15] and has been used as part of a joint correction scheme in [7]. However, an accurate estimation of the distortion near the resection margin is challenging due to the low spatial resolution of EPI, the heavy compression of voxels and the severe signal dropout that fades and blurs the resection margin and its neighbourhood in the b0-DWMRI image. A related approach is to acquire a pair of b0-DW-MRI images using opposite PE directions and thus exhibiting opposite distortions, and to estimate the distortion by recovering the mid-point image [1]. However, this presents an additional challenge of compensating between a heavy compression in one image and a heavy expansion in the pair image.

In this paper, we propose to simulate a field map from air-tissue segmentations generated using non-EPI 
T1-weighted $(\mathrm{T} 1 \mathrm{w})$ and $\mathrm{T} 2 \mathrm{w}$ iMRI images acquired as part of a clinical iMRI scanning protocol to provide an anatomically accurate, physically realistic and smooth prior for the above methods. Previously, Jenkinson et al. [13] developed a perturbation method to calculate a $B_{0}$ inhomogeneity field from air-tissue segmentation derived from computed tomography (CT) images. Poynton et al. [20] demonstrated that non-surgical T1w images can be segmented into air and tissue classes using a probabilistic $\mathrm{CT}$ atlas and reported that a subsequent application of the method [13] results in a close overall agreement between the acquired and simulated field maps. However, we observe that a probabilistic atlas is not suited to the segmentation of intraoperative iMRI images that contain air-filled craniotomy and resection areas with a shape specific to the surgery and the subject. Instead, we inform the air-tissue segmentation using priors derived from a synthetic CT image, which we compute from the preoperative $\mathrm{T} 1 \mathrm{w}$ image based on a database of MRI/CT pair templates. We subsequently feed the air-tissue segmentation into the method [13].

The field map simulation is evaluated by comparison with field maps acquired during iMRI guided ATLR neurosurgery for 12 cases. Our previous study presented at IPCAI 2014 has shown that the proposed method generates field maps in close agreement with the acquired field maps [16]. In this paper, we interpret the differences between the acquired and simulated field maps based on a quantitative evaluation of the effects of susceptibility correction using manually annotated anatomical landmarks and tensor fit error maps.

\section{Methods}

In Section 2.1 we introduce the method we used to correct EPI images in our study based on field maps and in Section 2.4.1 we outline the preprocessing step used for raw acquired field maps in order to enable the comparison of acquired and simulated field maps. In Section 2.2 we describe an air-tissue segmentation scheme used to bootstrap a field map simulation outlined in Section 2.3. An overview of the processing pipeline, needed to correct a DW-MRI image based on either an acquired or a simulated field map, is shown in Figure 1.

\subsection{Field map in terms of voxel displacement}

Let the magnetic field at point $\mathbf{x}$ be $B_{0}+\Delta B_{0}(\mathbf{x})[\mathrm{T}]$ where $B_{0}$ is the homogeneous field and $\Delta B_{0}(\mathbf{x})$ is the inhomogeneity field map, which can be equivalently expressed as $\gamma \Delta B_{0}(\mathbf{x})[\mathrm{rad} / \mathrm{s}]$ or $\frac{\gamma \Delta B_{0}(\mathbf{x})}{2 \pi}[\mathrm{Hz}]$. For the purposes of image correction, one is interested in the millimeter displacement along the phase encode direction that the inhomogeneity causes to an EPI image. The displacement can be calculated based on theory in $[14,11]$. Consider the acquisition of a single EPI slice with matrix size $N \times N$ and voxel dimensions $r_{\mathrm{FE}}$ in the frequency encode (FE) direction and $r_{\mathrm{PE}}$ in the phase encode (PE) direction, respectively. The EPI slice is reconstructed by the inverse Fourier transform of the MR signal. In the PE direction, the MR signal sampling rate is $\frac{N}{T_{\mathrm{RO}}}[\mathrm{Hz}]$, where $T_{\mathrm{RO}}$ is the readout time. The image bandwidth in the PE direction is $\frac{N}{N T_{\mathrm{RO}}}=\frac{1}{T_{\mathrm{RO}}}$ $[\mathrm{Hz} / \mathrm{pixel}]$ or $T_{\mathrm{RO}}[\mathrm{pixel} / \mathrm{Hz}]$. Since the $\mathrm{PE}$ gradient is used to encode position along the PE direction, the above offset corresponds to a distortion along the PE direction of size

$d_{\mathrm{PE}}(\mathbf{x})=\frac{\gamma \Delta B_{0}(\mathbf{x})}{2 \pi} T_{\mathrm{RO}} r_{\mathrm{PE}}$

in millimeters. The magnetic field $\Delta B_{0}(\mathbf{x})$ is either found by simulation or, in case of the acquired field map, can be derived from the phase evolution $\Delta \phi(\mathbf{x})$ [rad] of phase during the echo time difference $T_{\mathrm{ED}}$ :

$\Delta B_{0}(\mathbf{x})=\frac{\Delta \phi(\mathbf{x})}{\gamma T_{\mathrm{ED}}}$

A scalar displacement map was calculated using Equation 1 and was converted into a dense displacement field along the PE direction [7]. Identical displacement was applied to all the EPI images in each DW-MRI dataset by resampling the images with cubic spline interpolation using the resampling utility from the NiftyReg package [17].

\subsection{Air-tissue segmentation}

The magnetic susceptibility values for soft-tissue $(\approx$ $\left.-9.1 \times 10^{-6}\right)$ and bone $\left(\approx-11.4 \times 10^{-6}\right)$ are similar, but both are significantly different from that of air $\left(\approx 0.4 \times 10^{-6}\right)[20]$. Therefore, we needed a binary labeling of the head into tissue (which combines soft tissue and bone) and air.

For each subject, a segmentation was performed on the sum $(\mathrm{T} 1 \mathrm{w}+\mathrm{T} 2 \mathrm{w})$ of the intraoperative T1-weighted (T1w) and T2-weighted (T2w) MRI images, which were already co-registered, as the head was fixed during acquisitions. In T1w images, soft tissue with low free water content (e.g. the brain, muscles, fat) appears bright, while in $\mathrm{T} 2 \mathrm{w}$ images, the voxels with high free water content (such as CSF and the eyes) appear bright. Therefore, an accurate delineation of the soft-tissues can be obtained by summing the T1w and T2w MRI images. However, since bone and air have similar low 


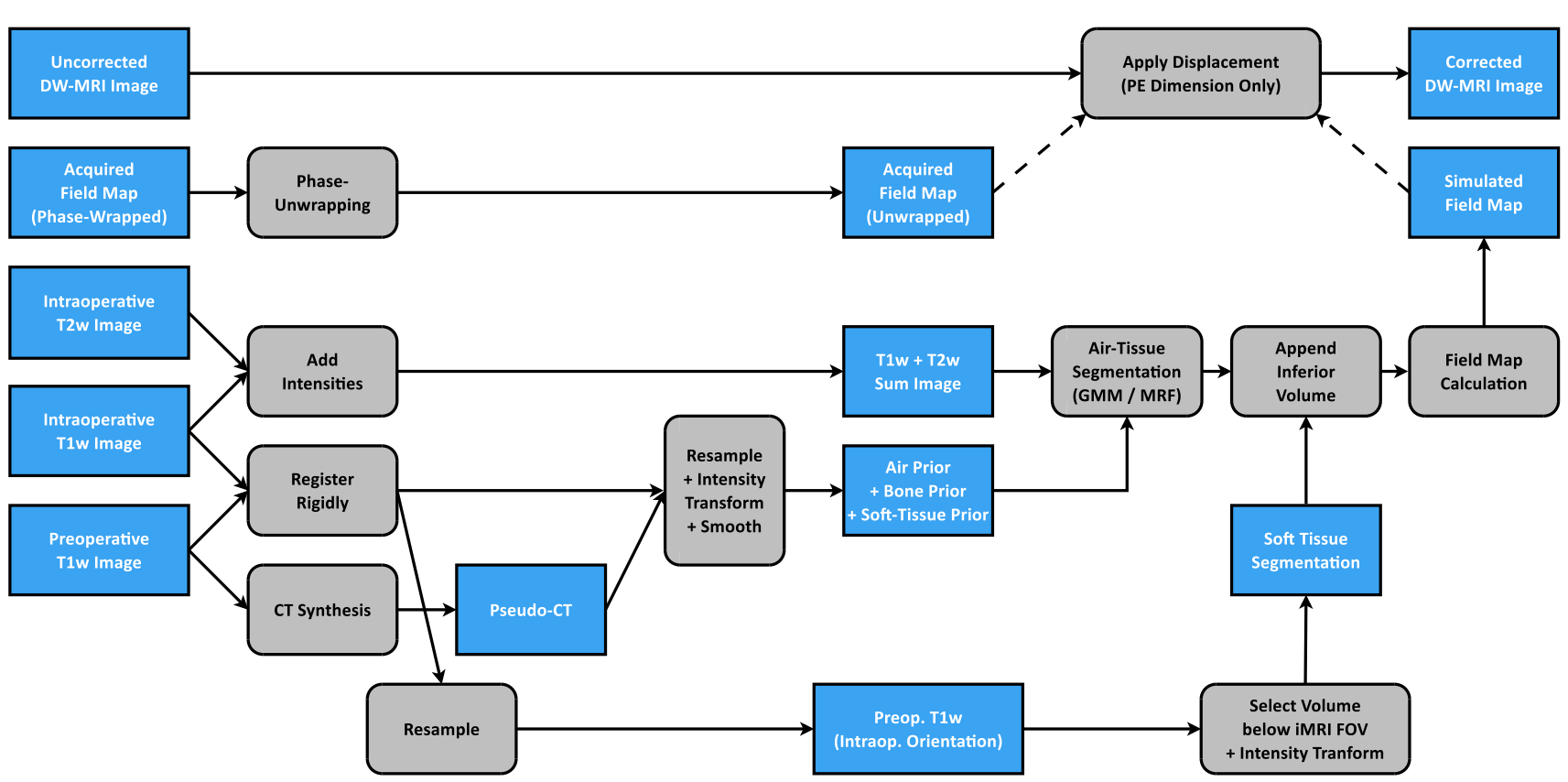

Fig. 1: The processing pipeline for correcting the susceptibility artefact in a DW-MRI image. The dashed lines represent the step into which either a phase-unwrapped acquired field map or a simulated field map can be fed. The entry points of the simulation are non-distorted preoperative $\mathrm{T} 1 \mathrm{w}$ and intraoperative $\mathrm{T} 1 \mathrm{w}$ and $\mathrm{T} 2 \mathrm{w}$ images.

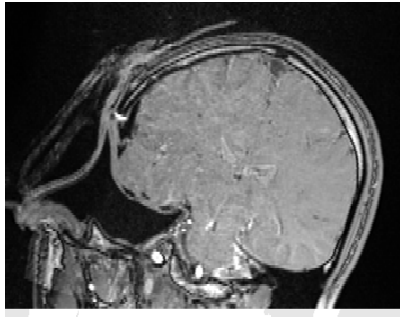

(a)

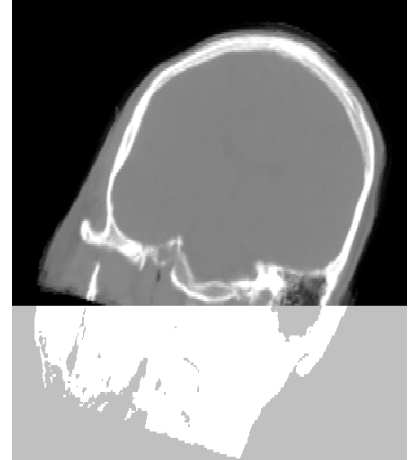

(b)

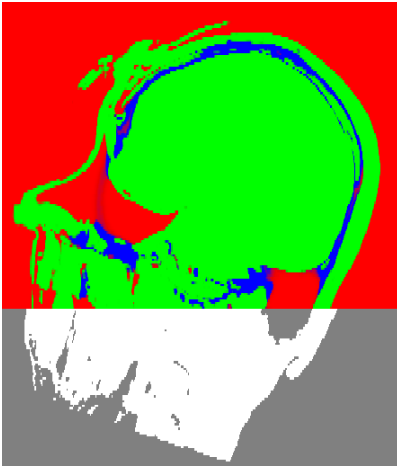

(c)

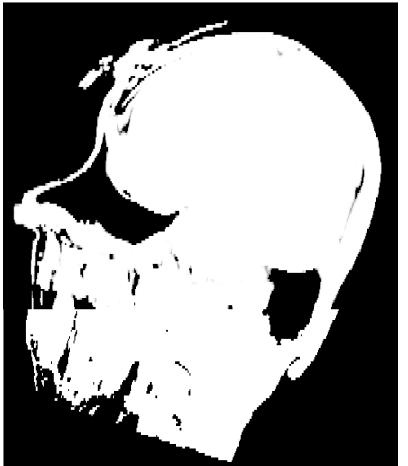

(d)

Fig. 2: Air-tissue segmentation. (a) Intraoperative T1w+T2w sum image. The section runs through a plane close to the anatomical coronal plane with the head at an angle due to intraoperative orientation. (b) An accompanying pseudo-CT (range -1000-1000 HU). (c) The result of the proposed segmentation within the intraoperative FOV (red for air, green for soft-tissue, blue for bone). (d) The final air-tissue segmentation (black for air, white for tissue). The appended inferior volume outside of the intraoperative FOV is an approximate air-tissue segmentation based on preoperative T1w MRI.

intensities in both $\mathrm{T} 1 \mathrm{w}$ and $\mathrm{T} 2 \mathrm{w}$ images, they are indistinguishable based on intensity alone. In order to distinguish between air and bone, we used a spatial prior probability map (spatial prior) associated with each class, which enables the segmentation of the $\mathrm{T} 1 \mathrm{w}+\mathrm{T} 2 \mathrm{w}$ image into three partial volume classes (air, soft-tissue and bone).
Air and bone have distinct attenuation properties, which makes CT images a good candidate for the spatial priors, as their intensities expressed in Hounsfield units (HU), are a linear transformation of the linear attenuation coefficients. However, CT images were not part of the preoperative planning imaging protocol. The attenuation information was derived from a pseudo-CT, 
synthesized from a preoperative $\mathrm{T} 1 \mathrm{w}$ image, as shown in Fig. 2(b).

\subsubsection{CT synthesis}

The CT synthesis method, developed by Burgos et al. $[2,3]$ relies on a pre-acquired set of aligned T1w MRI/CT image pairs from multiple subjects forming an MRI-CT database. Two gender-specific databases were used, both comprising 35 subjects part of an epilepsy study. To generate the CT from the preoperative T1w MRI image, each MRI image from the database was non-rigidly registered to the preoperative T1w MRI image. The CT images were then mapped using the same transformation to the preoperative $\mathrm{T} 1 \mathrm{w}$ MRI image. A local image similarity measure between the preoperative T1w MRI and the set of registered MRIs from the database was used as a surrogate of the underlying morphological similarity, under the assumption that if two MRIs are similar at a certain spatial location, the two CTs will also be similar at this location. Finally, the set of registered CTs was fused using a spatially varying weighted averaging, with weights derived from the similarity measure, generating a pseudo-CT.

\subsubsection{Constrained spatial priors}

To constrain the spatial priors to the probability range $0-1$, we used the sigmoid intensity transformation $\operatorname{sig}(I(\mathbf{x}))=1 /\left(1+\exp \left(\phi_{0}+\phi_{1} I(\mathbf{x})\right)\right)$, where $I(\mathbf{x})$ is the image intensity at voxel $\mathbf{x}$ and the constants $\phi_{0}$ and $\phi_{1}$ are chosen so as to transform a particular intensity range to the transitional interval 0.001-0.999. We established the soft-tissue prior by intensity-transforming the $\mathrm{T} 1 \mathrm{w}+\mathrm{T} 2 \mathrm{w}$ image intensity range 50-90 (mild gray).

The pseudo-CT, synthesised from the preoperative T1w MRI, was rigidly propagated to the intraoperative space. The advantage of using CT images is that the Hounsfield scale is quantitative, whereby air corresponds to $-1000 \mathrm{HU}$, soft-tissue ranges from 0 to $100 \mathrm{HU}$ and bone from 700 to $3000 \mathrm{HU}$. Using the sigmoid function, we let the air prior to vary from 0.999 to 0.001 for -1000-0 HU and we let the bone prior to vary from 0.001 to 0.999 for $100-700 \mathrm{HU}$.

The spatial priors had no biological basis in the areas altered by the surgery. We linearly reduced the air prior into a range $0.1-0.9$ to enforce the resection cavity to be segmented as air. We smoothed the spatial priors with a Gaussian kernel $(\sigma=2 \mathrm{~mm})$ to enforce the skull to be segmented as bone and the skull segment removed during the craniotomy to be segmented as air.

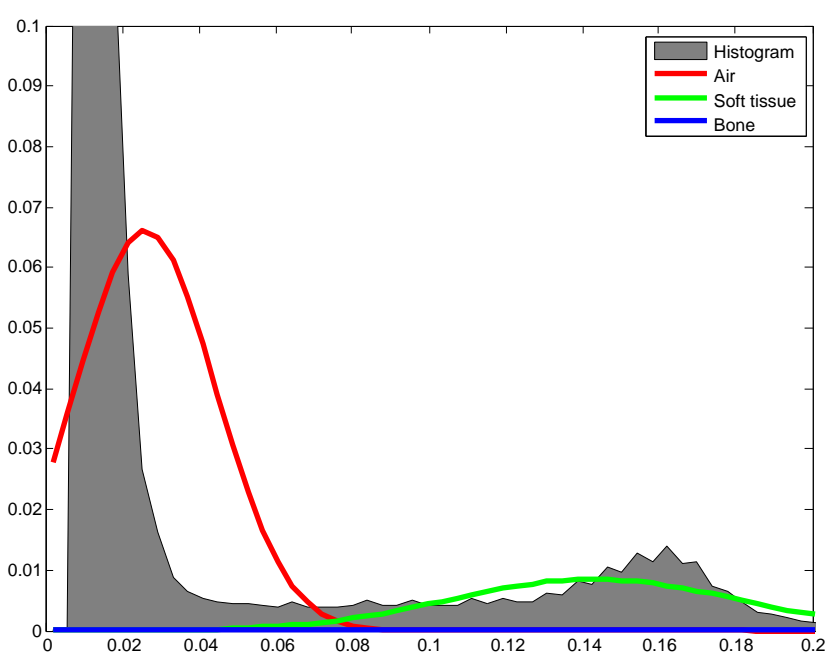

Fig. 3: Histogram (cropped for clarity of the figure) of the $\mathrm{T} 1 \mathrm{w}+\mathrm{T} 2 \mathrm{w}$ image in Fig. 2(a) and the fit of the GMM model for the segmentation in Fig. 2(a). The data come from intraoperative scans for subject \#3.

\subsubsection{Segmentation scheme}

The segmentation was obtained using the seg_EM tool from the NiftySeg package [4], which provides an intensity-based segmentation scheme based on the Gaussian Mixture Model (GMM) implemented using the Expectation-Maximization (EM) algorithm and a Markov Random Field (MRF) spatial regularization to provide a Maximum a Posteriori solution, as per Fig. 2(c). Fig. 3 shows the corresponding fitted GMM. We increased the MRF prior strenth $(\beta=1.0)$ to further enable a correct air segmentation in the craniotomy area.

iMRI and pseudo-CT fields of view (FOV) are largely limited to the cranial part of the head. The subsequent field map estimation step (Section 2.3) assumes that the voxels outside of the segmentation FOV are airfilled, which we observed to result in unrealistic field maps due to the virtual tissue cut-off below the cranium. We utilized the wide FOV of the rigidly registered preoperative $\mathrm{T} 1 \mathrm{w}$ and and appended a $100 \mathrm{~mm}$-deep volume inferiorly to the intraoperative FOV containing an approximate air-tissue segmentation obtained by thresholding the preoperative T1w (Fig. 2(d)).

\subsection{Field map estimation}

The field map estimation was performed using the tool b0calc from the FSL software package $[21,13]$, which models the first order perturbations of the main magnetic field. The susceptibility $\chi$ can be expanded as 
$\chi=\chi_{0}+\delta \chi_{1}$, where $\chi_{0}$ is the magnetic susceptibility of air, $\delta$ is the susceptibility difference between air and brain tissue and $\chi_{1}$ is a binary variable describing the tissue type. The first order perturbations of the z-component of the main magnetic field $\left(B_{z}^{1}\right)$ can be written in terms of the main magnetic field $\left(B_{z}^{0}\right)$ :

$B_{z}^{1}=\frac{\chi_{1}}{3+\chi_{0}} B_{z}^{0}-\frac{1}{1+\chi_{0}}\left(\left(\frac{\partial^{2} G}{\partial z^{2}}\right) *\left(\chi_{1} B_{z}^{0}\right)\right)$

where $G$ is the Green's function $G(x)=(4 \pi r)^{-1}$ and $r=\sqrt{x^{2}+y^{2}+z^{2}}$. Note that the expression is simplified considerably due to the fact that we only have a non-zero component $B_{z}^{0}$ in the longitudinal axis (zdirection) of the main magnetic field.

Equation (3) cannot be evaluated analytically for an arbitrary susceptibility distribution $\chi_{1}$. However, an analytical solution $H(\mathbf{x})$ exists for a single voxel of size $(a, b, c)$ centered at origin (with $\chi_{1}=1$ inside a voxel and $\chi_{1}=0$ outside of the voxel) for a constant field along the $\mathrm{z}$-axis and is given by:

$$
\begin{array}{r}
H(\mathbf{x})=\left(\frac{\partial^{2} G}{\partial z^{2}}\right) *\left(\chi_{1} B_{z}^{0}\right)= \\
\sum_{i, j, k \in(-1,1)}(i j k) F\left(x+\frac{i a}{2}, y+\frac{j b}{2}, z+\frac{k c}{2}\right)
\end{array}
$$

where $F(\mathbf{x})=\frac{1}{4 \pi} \arctan \left(\frac{x y}{z r}\right)$.

Due to the linearity of Equation (3), the shifted single voxel solutions $H\left(\mathbf{x}-\mathbf{x}^{\prime}\right)$ can be added together using a discrete convolution to compute the total field:

$B_{z}^{1}(\mathbf{x})=\sum_{\mathbf{x}^{\prime}} \chi_{1}\left(\mathbf{x}^{\prime}\right) H\left(\mathbf{x}-\mathbf{x}^{\prime}\right)$

where $\mathbf{x}^{\prime}$ are the voxel locations, $\chi_{1}\left(\mathbf{x}^{\prime}\right)$ is the voxelbased susceptibility map and $\mathbf{x}$ is the point where the field is evaluated. The discrete convolution can be implemented using the 3D Fast Fourier Transform (FFT).

Although this approach simulates the field distribution due to the main coil, it does not account for the decrease in inhomogeneity as effected by the roomtemperature shim coils, which are wound to form fields that follow first and second order spherical harmonics $S(\mathbf{x})=\left[x, y, z, z^{2}-\left(x^{2}+y^{2}\right) / 2, x z, y z, x^{2}-y^{2}, 2 x y\right](\mathbf{x})$, where $\mathbf{x}=\mathbf{0}$ at the magnet isocentre [10]. The field in the scanner becomes $B_{z}^{1}(\mathbf{x})-S \boldsymbol{\theta}$, where the coefficients $\boldsymbol{\theta}=\left[\theta_{1}, \theta_{2}, \ldots, \theta_{8}\right]^{T}$ are proportional to the currents in the shim coils, which are dynamically optimized by the scanner during image acquisition based on the field in the imaged volume [10]. In this simulation, we approximate the shim currents as a linear combination that minimizes the inhomogeneity field across the field of view, as used in [20]. We perform a least-squares fit of the spherical harmonics to determine $\hat{\boldsymbol{\theta}}=\operatorname{argmin}_{\boldsymbol{\theta}}\left(\left(B_{z}^{1}(\mathbf{x})-S \boldsymbol{\theta}\right)^{T}\left(B_{z}^{1}(\mathbf{x})-S \boldsymbol{\theta}\right)\right)$.

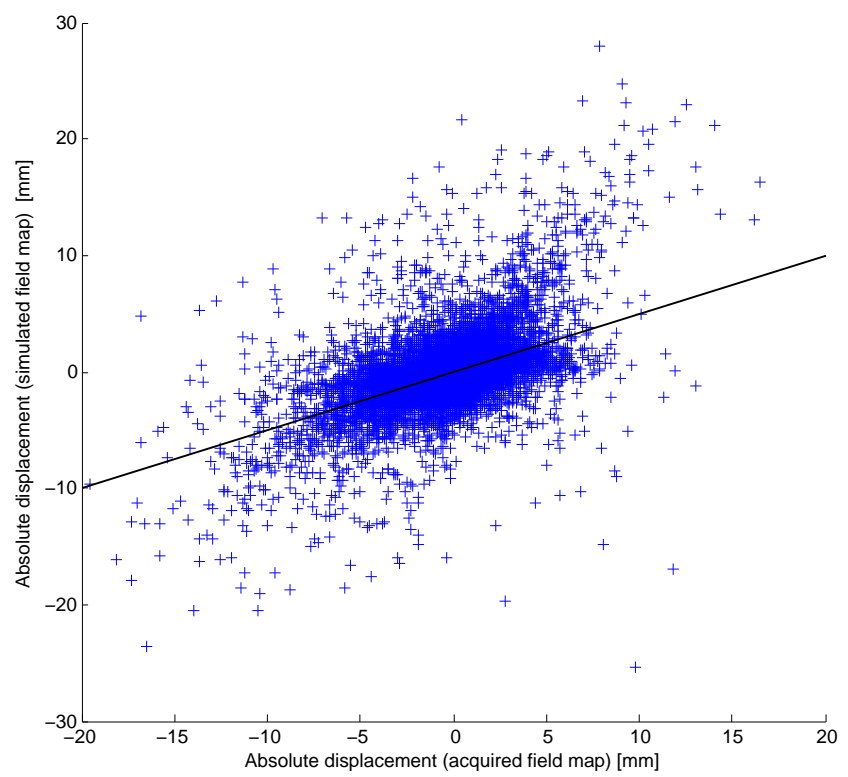

Fig. 4: Scatter plot of the acquired and the simulated field map in corresponding voxels inside the brain for subject \#3.

\subsection{Data acquisition}

The proposed algorithm was validated on 12 datasets that were acquired using interventional MRI during ATLR procedures. The study was approved by the National Hospital for Neurology and Neurosurgery and the UCL Institute of Neurology Joint Research Ethics Committee. Written informed consent was obtained from all participants. The images were acquired using a $1.5 \mathrm{~T}$ Espree MRI scanner (Siemens, Erlangen, Germany) designed for interventional procedures. The intraoperative protocol included a conventional T1-weighted FLASH image $(\mathrm{TR}=5.25 \mathrm{~ms}, \mathrm{TE}=2.5 \mathrm{~ms}$, flip angle $=$ $15^{\circ}, 0.547 \times 0.547 \times 1.25 \mathrm{~mm}$ grid of $512 \times 512 \times 176$ voxels) a conventional T2-weighted turbo spin echo image $(\mathrm{TR}=3200 \mathrm{~ms}, \mathrm{TE}=510 \mathrm{~ms}$, flip angle $=$ $120^{\circ}, 1.0 \times 1.0 \times 1.0 \mathrm{~mm}$ grid of $256 \times 256 \times 176$ voxels), and a DW-MRI dataset acquired using a single shot EPI sequence with GRAPPA-based parallel imaging (acceleration factor of $2,2.5 \times 2.5 \times 2.5 \mathrm{~mm}$ grid $\left(r_{\mathrm{PE}}=2.5 \mathrm{~mm}\right)$ of $84 \times 84 \times 49$ voxels, signal readout time $T_{\mathrm{RO}}=35.52 \mathrm{~ms}$ ) and a field map (see further below). The DW-MRI dataset consisted of set of 64 diffusion-weighted EPI images accompanied by a b0DW-MRI image. The GRAPPA imaging resulted in a halving of EPI echoes acquired in each readout but the readout time above is given nominally with the reduction factored in. 


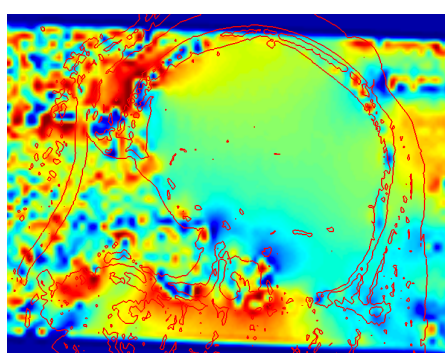

(a)

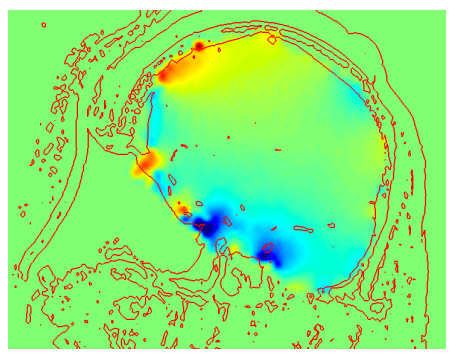

(d)

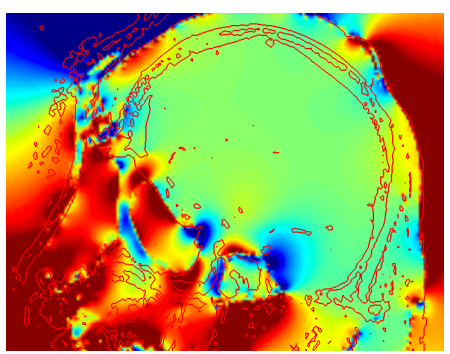

(g)

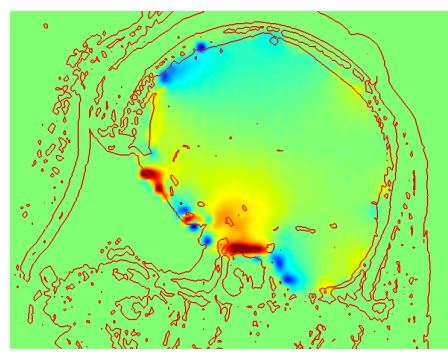

(j)

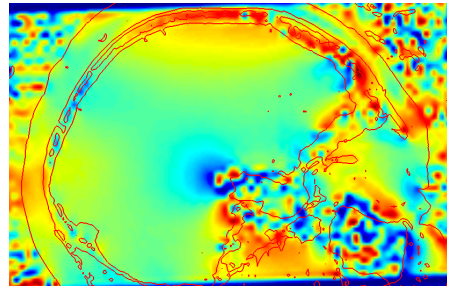

(b)

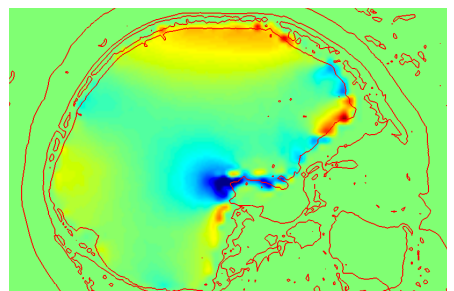

(e)

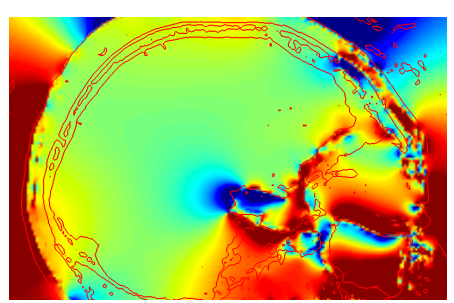

(h)

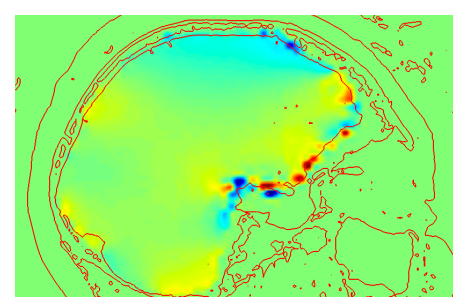

(k)

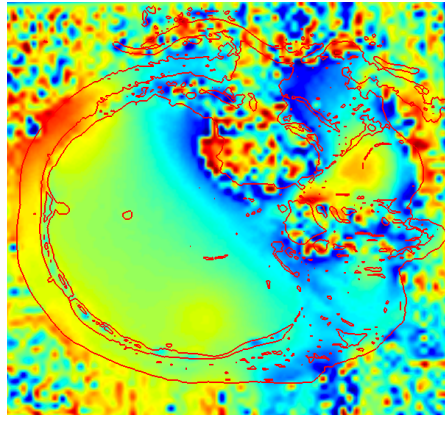

(c)

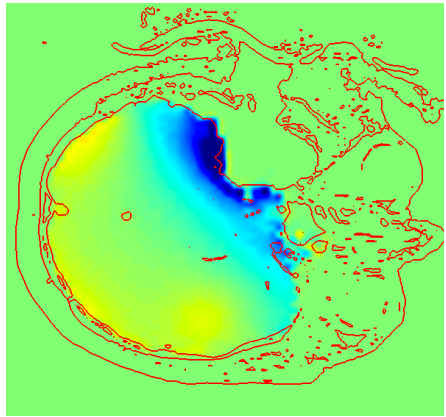

(f)

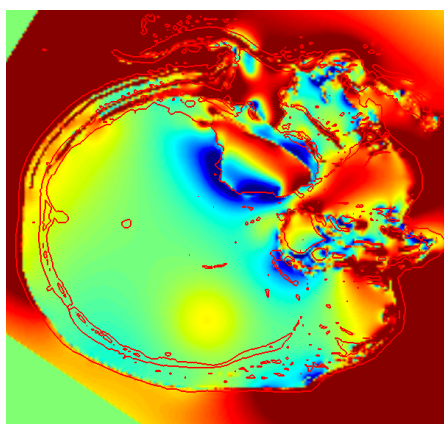

(i)

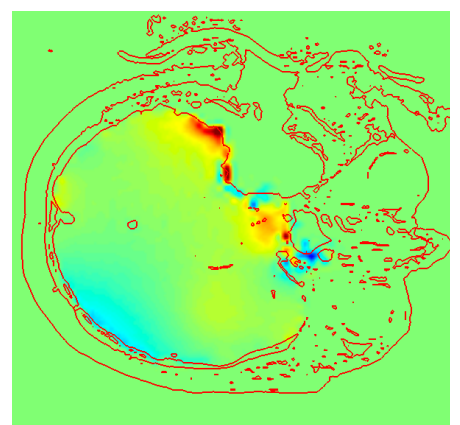

(1)

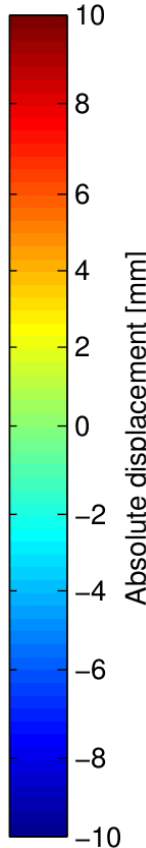

$-10$

6

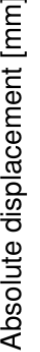

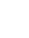




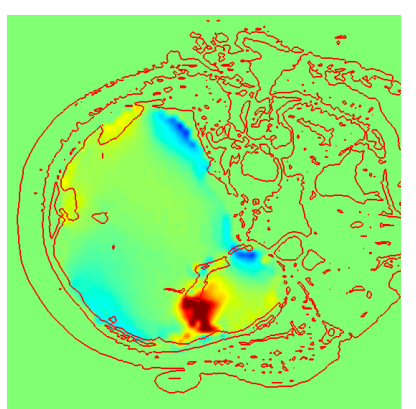

(a)

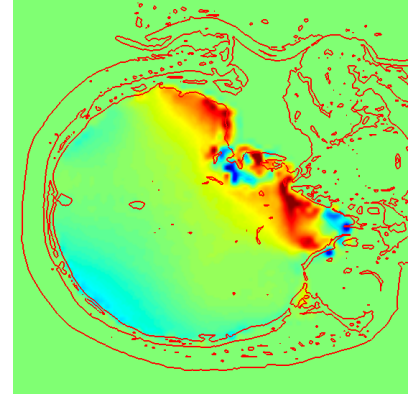

(b)

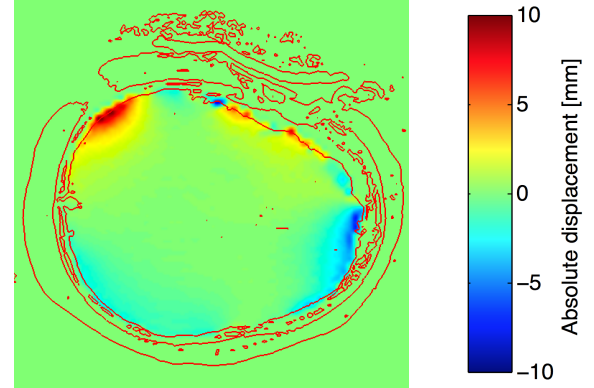

(c)

Fig. 6: Several axial slices through absolute difference between the simulated and phase-unwrapped acquired field maps, expressed as $\mathrm{mm}$ of displacement along the phase-encode direction shown for subject \#3. (a) Contralateral temporal lobe level. (b) Eye level. (c) Superior frontal and parietal lobe level.

\subsubsection{Field map acquisition}

The field maps were acquired using a gradient-recalled echo pulse sequence [14] $(2.91667 \times 2.91667 \times 2.9 \mathrm{~mm}$ grid of $72 \times 72 \times 43$ voxels, echo time difference of $\left.T_{\mathrm{ED}}=4.76 \mathrm{~ms}\right)$. The phase-unwrapping was performed using a method detailed in [7], which uses a Markov random field (MRF) formulation to enforce spatial smoothness in the estimated true field map. Since the recovered phase difference necessarily had an arbitrary constant component, we de-meaned map based on the voxels inside the brain mask. The resulting map $(\Delta \phi(\mathbf{x}))$ was converted using Eqs. 1 and 2 into displacement units.

\section{Experiments with clinical data and Results}

Since there is no direct way of measuring the true field maps in vivo, we compared the simulated field maps to the acquired field maps. We expressed the field maps in terms of mm of displacement along the PE direction (as per Equation 1). In all except one of the given DW-MRI datasets, the PE direction coincided with the anatomical anterior-posterior axis i.e. a positive field map value corresponded to displacement toward the posterior end of the image, while in one DW-MRI dataset, the PE direction was inverted. A scatter plot of the two types of maps for subject \#3 is shown in Fig. 4. We also calculated an absolute difference between the two types of maps (Fig. 5 and Fig. 6). We calculated statistics for the difference between the simulated and acquired field maps within the brain mask volume. The results for the 12 subjects are reported in Table $1^{1}$. For most of the brain, there is a close agreement, whereby the maps

\footnotetext{
1 The results reported in our original IPCAI 2014 paper showed slightly smaller displacements due to the voxel size being passed incorrectly.
}

\begin{tabular}{c|cc|cc}
\hline Subj. & \multicolumn{2}{|c|}{ Whole brain } & \multicolumn{2}{c}{ Disp. > 2.5 mm } \\
\hline & Mean ( std ) & P95 & Mean ( std ) & P95 \\
\hline 1 & $1.18(1.53)$ & 3.49 & $4.58(2.86)$ & 10.46 \\
2 & $1.06(1.24)$ & 3.17 & $4.10(2.01)$ & 7.91 \\
3 & $1.05(1.37)$ & 3.10 & $4.46(2.65)$ & 9.67 \\
4 & $1.10(1.53)$ & 3.48 & $4.71(3.04)$ & 10.42 \\
5 & $1.22(1.54)$ & 3.28 & $4.55(3.08)$ & 10.54 \\
6 & $1.02(1.36)$ & 3.00 & $4.57(2.76)$ & 10.26 \\
7 & $1.08(1.47)$ & 3.03 & $4.77(3.24)$ & 11.70 \\
8 & $0.89(1.35)$ & 2.99 & $4.53(2.74)$ & 10.05 \\
9 & $1.28(1.59)$ & 3.51 & $4.44(3.05)$ & 10.44 \\
10 & $1.34(1.72)$ & 4.15 & $4.60(2.73)$ & 10.23 \\
11 & $1.36(1.69)$ & 4.32 & $4.40(2.45)$ & 9.13 \\
12 & $1.19(1.46)$ & 3.62 & $4.35(2.49)$ & 9.09 \\
\hline Avg. & $1.15(1.49)$ & 3.43 & $4.51(2.76)$ & 9.99
\end{tabular}

Table 1: Absolute difference between the displacement (in $\mathrm{mm}$ ) in the phase encode dimension as per the proposed simulated field map and the acquired field map. The mean (std) and $95^{\text {th }}$ percentile values are reported for all the voxels in the brain and for those with the abs. difference above the voxel size $(2.5 \mathrm{~mm})$, respectively. The summary line lists the column averages.

differ by $1.15 \pm 1.49 \mathrm{~mm}$ on average $(3.43 \mathrm{~mm}$ in the $95^{\text {th }}$ percentile) for all the subjects within the brain volume. However, in some areas, there are larger disagreements and the differences follow a long-tailed distribution, whereby the the maps differ by $4.51 \pm 2.76 \mathrm{~mm}$ on average for all the subjects $\left(9.99 \mathrm{~mm}\right.$ in the $95^{\text {th }}$ percentile) in the voxels in which the absolute difference is above the voxel size $(2.5 \mathrm{~mm})$.

Since the acquired field maps are not the true field maps, we visually assessed the precision of EPI image correction in several areas of interest of the brain. The DW-MRI dataset for each subject was corrected as per Section 2.1 using the acquired field map and the proposed simulated field map, respectively. Examples for two subjects are shown in Fig. 7. We chose the b0-DW-MRI image from each DW-MRI dataset as 
the representative EPI image, because it contains visually identifiable landmarks. Since the conventional T2-weighted image has a similar tissue contrast profile as the b0-DW-MRI image, but has a higher resolution and is not affected by the susceptibility artefact, we used it as the ground truth image. We manually identified anatomical landmarks in the ground truth and susceptibility-distorted b0-DW-MRI images and measured the landmark misregistration in millimeters. We then propagated the landmarks from the distorted b0DW-MRI images using the acquired and simulated field maps, respectively, and measured the misregistration between the ground truth and the propagated landmarks. We only considered landmarks with reliable correspondences. Although in the T2-weighted images it was possible to identify features and localize them at image-resolution accuracy $\left(1 \mathrm{~mm}^{3}\right)$, conversely in the b0-DW-MRI images in areas of signal dropout or severe susceptibility distortion it was not possible to find reliable corresponding landmarks. In such cases, we collected nearest reliably identifiable landmarks.

We collected landmarks in 4 brain regions of interest (ROIs): (1) near the resection margin (posterior and superior from the resection cavity, 7-8 landmarks/subject, see Fig. 7(a)); (2) brain stem (due to clearly distinguishable margin and proximity to the resection cavity, 5 landmarks/subject, see Fig. 7(a)); (3) areas of high distortion in the proximity of the sites of the head-holder fixation pins (4-5 landmarks/subject, see Fig. 7(b)); (4) landmarks evenly distributed in the rest of the brain (6 landmarks/subject). Table 2 summarizes the landmark distances for the four ROIs. The titanium head fixation pins (ROI 3) are part of the head-holder (Noras, Hoechberg, Germany) and in their vicinity DW-MRI exhibits increased distortion, while $\mathrm{T} 1 \mathrm{w}$ and $\mathrm{T} 2 \mathrm{w}$ images exhibit signal dropout in the scalp.

We performed 3 one-tailed paired Student's $t$-tests for each ROI with the following alternative hypotheses: (a) landmark misregistration is smaller in images corrected using acquired field maps than in uncorrected images; (b) the same when correcting using simulated field maps; (c) landmark misregistration is smaller in images corrected using acquired field maps than in images corrected using simulated field maps. We set the significance level for a test regarding one ROI at $1.25 \%$ as per Bonferroni correction $(5 / 4=1.25 \%)$ in order to keep the family-wise error rate (FWER) below $5 \%$, where FWER is the probability of at least one null hypothesis being rejected due to chance alone. The results are reported in Table 3 .

However, landmark based validation is complicated due to non-repeatability of manual visual landmark lo- calization, as well as by the low resolution and poor quality of the b0-DW-MRI image and limited density of unambiguous landmarks. We corroborated our validation with an additional approach as in [7]. Since the DW-MRI datasets are ultimately used to estimate the presence of white matter areas in the brain, we evaluated the effect of susceptibility artefact correction on residual tensor fit errors.

The compression of the signals and the noise from adjacent voxels with unequal diffusion directions that occurs as a result of the highly non-linear local deformations arising from the susceptibility artefact contributes to the uncertainty in tensor fitting. [15] showed that a non-linear correction of DW-MRI datasets reduced tensor fit errors. Therefore, an accurate correction of susceptibility artefacts should reduce the tensor fit errors. We note that the modeling inadequacy of the tensor model, the limited voxel resolution, and the poor SNR that is especially severe in signal dropout areas, imply that even a perfect susceptibility correction cannot bring the tensor fit errors to zero. The normalized sum of square diffusion tensor fit errors $\left(\chi^{2}\right)$ is given by

$\chi^{2}=\frac{\sum_{i=1}^{N}\left(S_{m}-S_{f}\right)^{2}}{\sum_{i=1}^{N} S_{m}^{2}}$

where $N$ signals are fitted, and $S_{m}$ and $S_{f}$ are the measured and fitted signals respectively [19]. We note that the rotational component of the deforming field derived from the field map effectively rotates the diffusion gradient directions locally and thereby rotates the fitted diffusion tensor principal directions, but residual tensor fit errors are rotation-invariant as they are independent of the fitted tensor orientation. The diffusion tensors were reconstructed using dtifit from the FSL package [21], which also calculated the normalized tensor fit error maps. For the validation, tensor fitting was performed on the uncorrected DW-MRI datasets for the 12 subjects, on DW-MRI datasets with every of the 65 diffusion direction images individually corrected using the acquired field maps, and on DW-MRI datasets likewise corrected using the proposed simulated field maps, as shown in Fig. 8. The maps of normalized sum of square diffusion tensor fit errors were added up across the whole brain, as reported in Table 4. One-tailed paired Student's t-tests showed a statistically significant reduction for DW-MRI datasets corrected using both the acquired field maps $\left(p=3.43 \times 10^{-8}\right.$, confidence interval lower bound at $5 \%$ significance level: 0.72 $\mathrm{mm})$ and the simulated field maps $\left(p=3.56 \times 10^{-8}, \mathrm{CI}\right.$ : $0.41 \mathrm{~mm}$ ). The said reduction was significantly larger for DW-MRI datasets corrected using the acquired field maps than for those corrected using the simulated field maps $\left(p=2.32 \times 10^{-6}, \mathrm{CI}: 0.28 \mathrm{~mm}\right)$. 


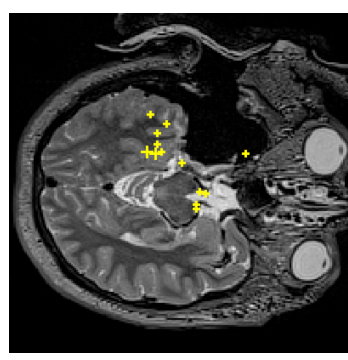

(a)

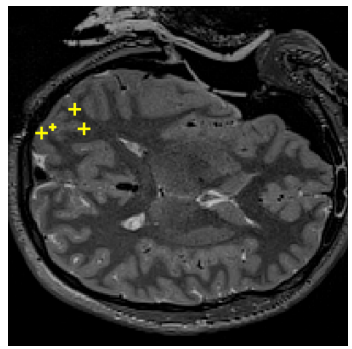

(e)

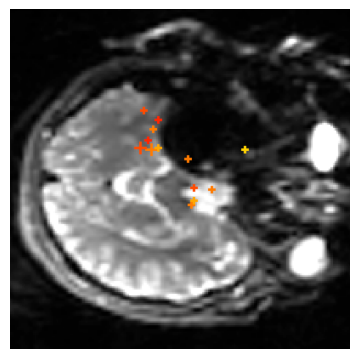

(b)

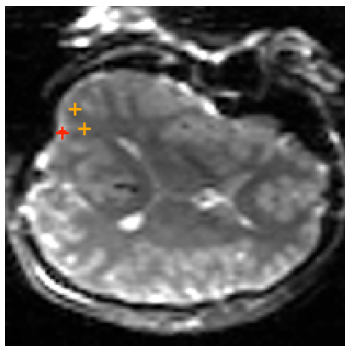

(f)

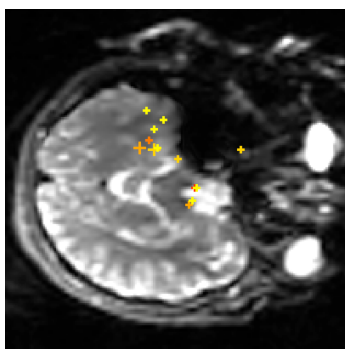

(c)

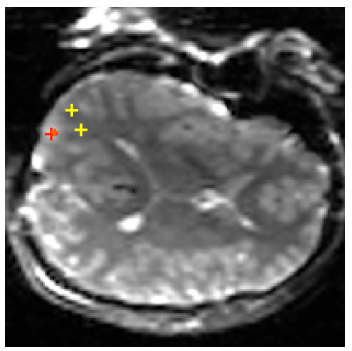

(g)

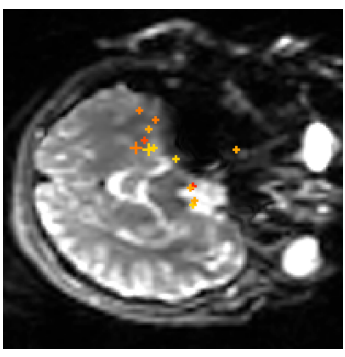

(d)

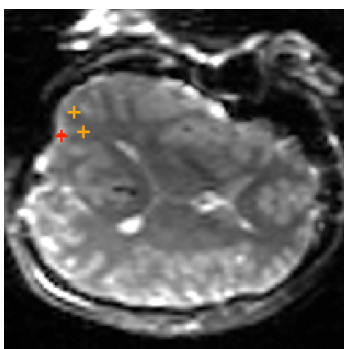

(h)

Fig. 7: Landmark based evaluation of susceptibility artefact correction. Manually located anatomical landmarks are shown color -coded based on distance from ground truth position (large cross: in-slice, small cross: projection of landmark onto the shown slice). (a,e) Intraoperative T2-weighted image unaffected by the distortion (ground truth image for landmarks). (b,f) An uncorrected susceptibility-distorted b0-DW-MRI image. (c,g) The b0-DWMRI image corrected using the acquired field map. $(\mathrm{d}, \mathrm{h})$ The b0-DW-MRI image corrected using the proposed simulated field map. The top row (a-d) shows the vicinity of the resection cavity (ROI 1 in Table 2) and brain stem (ROI 2 in Table 2) for subject \#3 with resection in the right hemisphere. The bottom row (e-h) shows the area of strong brain surface distortion (ROI 3 in Table 2) for subject \#4.

\begin{tabular}{c|ccc||ccc||ccc||ccc}
\hline Subj. & \multicolumn{3}{|c||}{ ROI 1 } & \multicolumn{3}{c||}{ ROI 2 } & \multicolumn{3}{c||}{ ROI 3 } & \multicolumn{3}{c}{ ROI 4 } \\
\hline & Unc. & AFM & SFM & Unc. & AFM & SFM & Unc. & AFM & SFM & Unc. & AFM & SFM \\
\hline 1 & 2.80 & 0.40 & 1.80 & 4.86 & 3.26 & 4.26 & 10.75 & 4.00 & 8.75 & 2.80 & 1.00 & 2.20 \\
2 & 2.33 & 1.17 & 1.33 & 4.20 & 2.62 & 4.80 & 4.00 & 1.45 & 3.00 & 1.67 & 1.00 & 2.01 \\
3 & 3.50 & 0.88 & 2.37 & 3.00 & 1.52 & 2.18 & 6.00 & 2.40 & 5.60 & 4.75 & 3.86 & 4.89 \\
4 & 3.51 & 1.77 & 1.90 & 3.40 & 1.60 & 1.80 & 6.07 & 2.65 & 6.07 & 1.94 & 0.88 & 1.21 \\
5 & 2.04 & 0.79 & 0.92 & 3.05 & 0.68 & 1.20 & 8.00 & 4.75 & 7.75 & 2.17 & 0.67 & 2.00 \\
6 & 2.56 & 1.51 & 1.81 & 2.40 & 1.45 & 1.10 & 8.00 & 4.25 & 6.75 & 2.00 & 1.00 & 1.50 \\
7 & 2.83 & 0.67 & 1.50 & 2.95 & 1.80 & 1.55 & 8.75 & 5.25 & 7.50 & 2.00 & 0.50 & 1.50 \\
8 & 3.43 & 1.28 & 2.00 & 2.78 & 1.20 & 1.60 & 8.75 & 5.00 & 5.75 & 0.83 & 0.84 & 1.00 \\
9 & 6.10 & 4.02 & 5.56 & 1.59 & 0.64 & 1.00 & 3.60 & 1.50 & 3.40 & 9.02 & 7.99 & 8.26 \\
10 & 2.48 & 1.56 & 2.27 & 3.60 & 1.20 & 2.00 & 5.60 & 2.80 & 5.00 & 2.67 & 0.67 & 1.83 \\
11 & 6.88 & 2.20 & 4.89 & 2.20 & 0.40 & 0.60 & 2.21 & 0.75 & 2.75 & 2.33 & 1.33 & 2.33 \\
12 & 3.07 & 1.21 & 2.25 & 4.00 & 1.74 & 1.60 & 6.35 & 3.81 & 6.75 & 1.50 & 0.84 & 1.17 \\
\hline Avg. & 3.46 & 1.46 & 2.38 & 3.17 & 1.51 & 1.97 & 6.51 & 3.22 & 5.76 & 2.81 & 1.72 & 2.49
\end{tabular}

Table 2: Misregistration (in $\mathrm{mm}$ ) between the ground truth landmarks and the landmarks in the uncorrected b0DW-MRI images (Unc.) and those corrected using acquired field maps (AFM) and simulated field maps (SFM). The results are reported for 4 ROIs: (1) near resection margin, (2) brain stem, (3) head-holder fixation pins, (4) rest of the brain. The summary line contains the column averages. 


\begin{tabular}{ccccc}
\hline Misregistration & ROI 1 & ROI 2 & ROI 3 & ROI 4 \\
\hline (a) Corr./AFM $<$ Unc. & $1.34 \times 10^{-5}$ & $8.91 \times 10^{-8}$ & $1.37 \times 10^{-6}$ & $1.26 \times 10^{-5}$ \\
& $(1.25)$ & $(1.29)$ & $(2.31)$ & $(0.68)$ \\
\hline (b) Corr./SFM < Unc. & $4.34 \times 10^{-6}$ & $1.10 \times 10^{-4}$ & $1.34 \times 10^{-2}$ & $1.02 \times 10^{-2}$ \\
& $(0.72)$ & $(0.62)$ & $(-0.012)$ & $(0.13)$ \\
\hline (c) Corr./AFM < Corr./SFM & $7.16 \times 10^{-4}$ & $1.88 \times 10^{-2}$ & $1.66 \times 10^{-6}$ & $2.58 \times 10^{-5}$ \\
& $(0.36)$ & $(-0.045)$ & $(1.77)$ & $(0.46)$
\end{tabular}

Table 3: Results of one-tailed Student's t-test of landmark misregistration reduction between uncorrected (Unc.) images and those corrected (Corr.) using acquired (AFM) and simulated (SFM) field maps ( $p$-value and lower bound of the CI at $1.25 \%$ significance level in $\mathrm{mm}$ ).

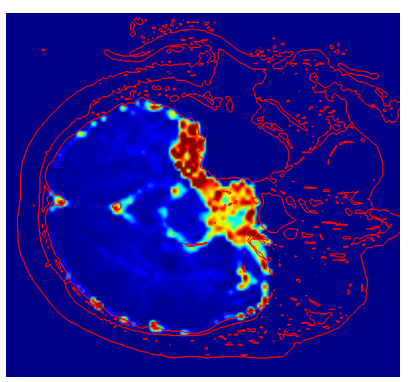

(a)

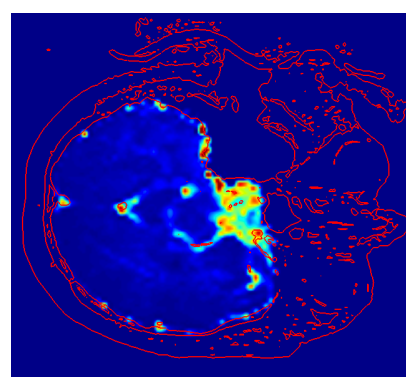

(b)

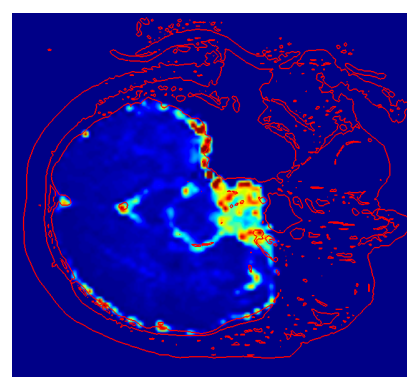

(c)

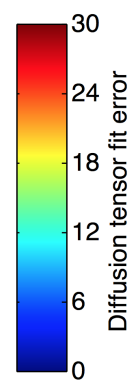$$
\text { . }
$$

Fig. 8: Map of normalized sum of square diffusion tensor fit errors shown for subject \#3 calculated from (a) an uncorrected example DW-MRI dataset, (b) the DW-MRI dataset corrected using an acquired field map and (c) a simulated field map.

\begin{tabular}{cccc}
\hline Subj. & Unc. & AFM & SFM \\
\hline 1 & $5.16(7.61)$ & $4.07(5.61)$ & $4.60(6.61)$ \\
2 & $3.43(5.59)$ & $2.79(4.21)$ & $3.10(5.02)$ \\
3 & $3.92(6.79)$ & $3.09(5.19)$ & $3.29(5.57)$ \\
4 & $4.96(7.58)$ & $4.16(6.17)$ & $4.47(6.70)$ \\
5 & $5.06(6.98)$ & $4.01(5.30)$ & $4.50(6.15)$ \\
6 & $3.48(6.57)$ & $2.72(4.81)$ & $3.02(5.42)$ \\
7 & $3.86(6.86)$ & $3.06(5.43)$ & $3.39(6.08)$ \\
8 & $4.27(6.40)$ & $3.60(5.16)$ & $3.82(5.55)$ \\
9 & $5.39(7.18)$ & $4.37(5.68)$ & $4.72(6.20)$ \\
10 & $3.33(6.00)$ & $2.65(4.67)$ & $3.07(5.60)$ \\
11 & $5.86(7.74)$ & $4.60(5.89)$ & $5.27(6.85)$ \\
12 & $3.28(5.55)$ & $2.84(4.83)$ & $2.99(5.03)$ \\
\hline Avg. & $4.33(6.74)$ & $3.50(5.25)$ & $3.85(5.90)$
\end{tabular}

Table 4: Mean (std) of normalized sum of square diffusion tensor fit errors summed up across the whole brain volume. The results are reported for the uncorrected (Unc.) DW-MRI datasets and those corrected using the acquired (AFM) and the simulated field maps (SFM). The summary line contains the column averages.

\section{Discussion}

We found that the GMM model classified $\mathrm{T} 1 \mathrm{w}+\mathrm{T} 2 \mathrm{w}$ voxels representing the CSF and the eyes as soft-tissue, which is correct for susceptibility modelling. The main challenge for an accurate air-tissue segmentation was the ambiguity between the air and bone voxels. The use of a strong MRF and the smoothing of the air prior ensured that skull voxels were generally correctly classified as bone. The strong MRF also forced skull bone marrow, bright in $\mathrm{T} 1 \mathrm{w}+\mathrm{T} 2 \mathrm{w}$, to be included in the bone class, which is acceptable due to the virtually identical susceptibility. The distribution for the bone class in the GMM model received a low weight due to the sparsity of the bone voxels in the prior (Fig. 3). In future work, the $\mathrm{T} 1 \mathrm{w}+\mathrm{T} 2 \mathrm{w}$ image could be replaced by multimodal segmentation.

The average difference found between the acquired and simulated field maps of $1.15 \pm 1.49 \mathrm{~mm}$ for all subjects within the brain volume (in terms of displacement along the PE direction) is less than the voxel size of the DW-MRI dataset $(2.5 \mathrm{~mm}$, typical for DW-MRI datasets) and has to be considered with respect to the desired resection accuracy, which is patient and surgeon specific and difficult to define. We believe that the observed near $1 \mathrm{~mm}$ resection accuracy in areas of low field map difference is clinically useful. However, there are areas of difference between the two types of field maps.

In the absence of ground truth field maps, we used landmark misregistration and sum of diffusion tensor fit errors as surrogate measures for statistical evaluation of susceptibility distortion correction using acquired and simulated field maps. The diffusion tensor fit errors based results indicate that both types of field 
maps facilitate DW-MRI dataset correction, although the acquired field maps facilitate a better correction compared to the simulated ones (Table 4). The landmark based results indicate that the acquired field maps facilitate landmark correction in all ROIs, while the simulated field maps facilitate significant correction in all ROIs except in the regions of high DW-MRI distortion near the head-holder fixation pins (second row, ROI 3, in Table 3). Also, in all ROIs except near the brain stem the results due to acquired field maps are better than due the simulated field maps (third row, ROI 2, in Table 3).

We attempt to interpret the differences between the field maps in specific areas. We observe that the simulated field maps are more positive in the immediate vicinity (i.e. up to $1 \mathrm{~mm}$ ) from the resection margin, as shown in Fig. $5(\mathrm{j}-\mathrm{l})$, i.e. the simulated field maps denote a further displacement along the PE direction than the acquired field maps. This area is challenging to evaluate using both landmark based and tensor fit error based approach due to the heavy noise in the DW-MRI data. The landmark based evaluation in the area up to approx. $10 \mathrm{~mm}$ from the resection margin (ROI 1) and in the nearby brain stem (ROI 2) (Fig. 7, a-d) is more conclusive and indicates that the acquired field maps provide superior DW-MRI correction in those regions. We found that the simulated field maps tend to co-vary with the acquired field maps in this region (Fig. 5, ai). We also observe that under the head-holder fixation pins (ROI 3), the simulated field maps are more positive than the acquired field maps. In these regions (Fig. 7, $\mathrm{e}-\mathrm{h}$ ) we found during landmark-based evaluation that only the acquired field maps provide significant EPI correction, and hence the simulated field maps are overestimated. This is likely due to the susceptibility of titanium from which the pins are manufactured. The problem is compounded by the fact that the resulting signal dropout is strong enough to appear in the scalp below the pins in $\mathrm{T} 1 \mathrm{w}$ and $\mathrm{T} 2 \mathrm{w}$ images, which leads to the scalp below the pins to be missegmented as air. We envisage that a separate study of the influence of the titanium pins, for instance based on simple digital phantoms inserted into an existing air-tissue segmentation, would reveal useful insight into the influence of the titanium pins.

We finally observe that near the air-filled petrous part of the temporal bone in both hemispheres and anteriorly in the frontal lobe (superior to the paranasal sinuses) the simulated field maps tend to form regions that are up to $10 \mathrm{~mm}$ more positive than the acquired field maps, as shown in Fig. 6, because the proposed segmentation overestimates the size of the air-filled cavities due to the relaxation of the air prior that we found as necessary to avoid a missegmentation of the craniotomy as bone. This limitation could be overcome if a robust automated approach for the segmentation of the resection cavity and the craniotomy area was available, such as based on active contours [23].

We also note that the simple method to estimate the room-temperature shim coil currents that we employed (as per Section 2.3) is unlikely to faithfully reproduce the dynamic optimization implemented by the iMRI scanner and potentially dependent on specific configuration e.g. shim coil current limits [10]. Such detailed entries are not part of the DICOM standard but we envisage to investigate the manufacturer specific subheader for this purpose.

The time to apply the field maps to a DW-MRI dataset is several seconds. The computational time to phase-unwrap the raw acquired field map is below 1 minute. Conversely, the computational time required to simulate the field map at the full T1-weighted iMRI resolution, in the current unaccelerated implementation, is approx. 1 hour (Intel Core i5 @ $3.30 \mathrm{GHz}$ ), out of which the air-tissue segmentation takes approx. 2 minutes. The time given assumes that the pseudo$\mathrm{CT}$ is precomputed or a real CT is imaged prior to the surgery and hence can be discounted from the intraoperative time. Although the simulation currently clearly takes too much time for intra-operative use, most of it is dominated by FFT (Section 2.3) that lends itself easily to GPU acceleration.

\section{Conclusion}

We generated simulated field maps in a 12-subject iMRI ALTR study that facilitate a statistically significant correction of susceptibility artefacts in EPI datasets near the resection cavity. We achieved a close agreement of the simulated field maps to the acquired field maps. Although we found that the acquired field maps facilitate statistically more accurate susceptibility artefact correction in all brain regions, the observed close agreement is very encouraging and invites a further study. We found that the reliability of automated air-tissue segmentation is key to accurate intraoperative field map simulations. We suggest a further study into the feasibility of including digital phantoms of the titanium head fixation pins into the automated simulation.

The voxel-based field calculation approach works with arbitrary segmentations and in principle allows field map simulations applied to iMRI guided resective surgery in other parts of the brain than the temporal lobe investigated in our study. While in our study DW-MRI was the only EPI dataset available, functional MRI (fMRI) imaging, which measures brain activity 
in real time and has been proposed for intraoperative use [9], is also based on EPI [11] and hence expands the range of applications that would benefit from accurate simulated field maps.

\section{Conflict of Interest}

The authors declare that they have no conflict of interest.

\section{References}

1. Andersson, J.L., Skare, S., Ashburner, J.: How to correct susceptibility distortions in spin-echo echo-planar images: application to diffusion tensor imaging. Neuroimage $\mathbf{2 0}(2), 870-888$ (2003)

2. Burgos, N., Cardoso, M.J., Modat, M., Pedemonte, S., Dickson, J., Barnes, A., Duncan, J.S., Atkinson, D., Arridge, S.R., Hutton, B.F., Ourselin, S.: Attenuation Correction Synthesis for Hybrid PETMR Scanners. In: Medical Image Computing and Computer-Assisted Intervention-MICCAI 2013, pp. 147154. Springer (2013)

3. Burgos, N., Cardoso, M.J., Thielemans, K., Modat, M., Pedemonte, S., Dickson, J., Barnes, A., Ahmed, R., Mahoney, C.J., Schott, J.M., Duncan, J.S., Atkinson, D., Arridge, S.R., Hutton, B.F., Ourselin, S.: Attenuation Correction Synthesis for Hybrid PET-MR Scanners: Application to Brain Studies. Medical Imaging, IEEE Transactions on (In print.)

4. Cardoso, M.J., Clarkson, M.J., Ridgway, G.R., Modat, M., Fox, N.C., Ourselin, S.: Improved Maximum a Posteriori Cortical Segmentation by Iterative Relaxation of Priors. In: Medical Image Computing and Computer-Assisted Intervention-MICCAI 2009, pp. 441449. Springer (2009)

5. Chen, X., Weigel, D., Ganslandt, O., Buchfelder, M., Nimsky, C.: Prediction of visual field deficits by diffusion tensor imaging in temporal lobe epilepsy surgery. Neuroimage 45(2), 286-297 (2009)

6. Clare, S., Evans, J., Jezzard, P.: Requirements for Room Temperature Shimming of the Human Brain. Magnetic resonance in medicine 55(1), 210-214 (2006)

7. Daga, P., Pendse, T., Modat, M., White, M., Mancini, L., Winston, G., McEvoy, A.W., Thornton, J., Yousry, T., Drobnjak, I., Duncan, J.S., Ourselin, S.: Susceptibility Artefact Correction using Dynamic Graph Cuts: Application to Neurosurgery. Medical Image Analysis 18(7), 1132-1142 (2014)

8. Daga, P., Winston, G., Modat, M., White, M., Mancini, L., Cardoso, M.J., Symms, M., Stretton, J., McEvoy, A.W., Thornton, J., Micallef, C., Yousry, T., Hawkes, D.J., Duncan, J.S., Ourselin, S.: Accurate Localization of Optic Radiation during Neurosurgery in an Interventional MRI Suite. Medical Imaging, IEEE Transactions on 31(4), 882-891 (2012)

9. Gasser, T., Ganslandt, O., Sandalcioglu, E., Stolke, D., Fahlbusch, R., Nimsky, C.: Intraoperative functional MRI: implementation and preliminary experience. Neuroimage 26(3), 685-693 (2005)
10. Gruetter, R., Boesch, C.: Fast, Noniterative Shimming of Spatially Localized Signals. In Vivo Analysis of the Magnetic Field along Axes. Journal of Magnetic Resonance (1969) 96(2), 323-334 (1992)

11. Hutton, C., Bork, A., Josephs, O., Deichmann, R., Ashburner, J., Turner, R.: Image Distortion Correction in fMRI: a Quantitative Evaluation. Neuroimage 16(1), 217-240 (2002)

12. Jenkinson, M.: Fast, automated, n-dimensional phaseunwrapping algorithm. Magnetic resonance in medicine 49(1), 193-197 (2003)

13. Jenkinson, M., Wilson, J.L., Jezzard, P.: Perturbation Method for Magnetic Field Calculations of Nonconductive Objects. Magnetic resonance in medicine 52(3), 471477 (2004)

14. Jezzard, P., Balaban, R.S.: Correction for Geometric Distortion in Echo Planar Images from B0 Field Variations. Magnetic resonance in medicine 34(1), 65-73 (1995)

15. Kim, D.J., Park, H.J., Kang, K.W., Shin, Y.W., Kim, J.J., Moon, W.J., Chung, E.C., Kim, I.Y., Kwon, J.S., Kim, S.I.: How does distortion correction correlate with anisotropic indices? a diffusion tensor imaging study. Magnetic resonance imaging 24(10), 1369-1376 (2006)

16. Kochan, M., Daga, P., Burgos, N., White, M., Cardoso, M.J., Mancini, L., Winston, G.P., McEvoy, A.W., Thornton, J., Yousry, T., Duncan, J.S., Stoyanov, D., Ourselin, S.: Simulated Field Maps: Toward Improved Susceptibility Artefact Correction in Interventional MRI. In: Information Processing in Computer-Assisted Interventions, pp. 226-235. Springer (2014)

17. Modat, M., Ridgway, G.R., Taylor, Z.A., Lehmann, M., Barnes, J., Hawkes, D.J., Fox, N.C., Ourselin, S.: Fast free-form deformation using graphics processing units. Computer methods and programs in biomedicine 98(3), 278-284 (2010)

18. Nilsson, D., Starck, G., Ljungberg, M., Ribbelin, S., Jönsson, L., Malmgren, K., Rydenhag, B.: Intersubject variability in the anterior extent of the optic radiation assessed by tractography. Epilepsy research 77(1), 11-16 (2007)

19. Papadakis, N.G., Martin, K.M., Mustafa, M.H., Wilkinson, I.D., Griffiths, P.D., Huang, C.L.H., Woodruff, P.W.: Study of the effect of CSF suppression on white matter diffusion anisotropy mapping of healthy human brain. Magnetic resonance in medicine 48(2), 394-398 (2002)

20. Poynton, C., Jenkinson, M., Wells III, W.: Atlas-Based Improved Prediction of Magnetic Field Inhomogeneity for Distortion Correction of EPI Data. In: Medical Image Computing and Computer-Assisted InterventionMICCAI 2009, pp. 951-959. Springer (2009)

21. Smith, S.M., Jenkinson, M., Woolrich, M.W., Beckmann, C.F., Behrens, T.E., Johansen-Berg, H., Bannister, P.R., De Luca, M., Drobnjak, I., Flitney, D.E., Niazya, R.K., Saundersa, J., Vickersa, J., Zhanga, Y., De Stefano, N., Brady, J.M., Matthews, P.M.: Advances in functional and structural MR image analysis and implementation as FSL. Neuroimage 23(Supplement 1), S208-S219 (2004)

22. Wiebe, S., Blume, W.T., Girvin, J.P., Eliasziw, M.: A randomized, controlled trial of surgery for temporal-lobe epilepsy. New England Journal of Medicine 345(5), 311318 (2001)

23. Xu, C., Prince, J.L.: Snakes, shapes, and gradient vector flow. Image Processing, IEEE Transactions on 7(3), 359369 (1998) 